\title{
Kernos
}

Revue internationale et pluridisciplinaire de religion grecque antique

17 | 2004

Varia

\section{Les artistes de l'épopée homérique : les femmes, le dieu, et le poète}

\section{Danièle Aubriot-Sévin}

\section{(2) OpenEdition \\ Journals}

\section{Édition électronique}

URL : http://journals.openedition.org/kernos/1401

DOI : 10.4000/kernos. 1401

ISSN : 2034-7871

\section{Éditeur}

Centre international d'étude de la religion grecque antique

\section{Édition imprimée}

Date de publication : 1 janvier 2004

ISSN : 0776-3824

\section{Référence électronique}

Danièle Aubriot-Sévin, «Les artistes de l'épopée homérique : les femmes, le dieu, et le poète », Kernos [En ligne], 17 | 2004, mis en ligne le 22 avril 2011, consulté le 01 mai 2019. URL : http:// journals.openedition.org/kernos/1401; DOI : 10.4000/kernos.1401 


\section{Les artistes de l'épopée homérique : les femmes, le dieu, et le poète'}

La question des représentations artistiques chez Homère est vaste ${ }^{2}$. Je me bornerai ici à explorer en quoi elles peuvent nous renseigner sur le sens des épopées et sur' une éventuelle correspondance entre l'œuvre du poète et celle du dieu. Cette étude trouve son origine dans une réflexion sur les rapports entre la «description » et la narration", et part du constat d'une sorte de chassé-croisé (qui se retrouve dans les deux épopées), entre des représentations ciselées dans le métal, et des broderies ou tissages figurés sur ou dans l'étoffe. Les premières sont surtout le fait d'Héphaïstos, les secondes relèvent du travail des femmes. Il m'apparaît que ces représentations artistiques sont, les unes et les autres, reliées aux modes de narration employés dans chacune des deux épopées homériques, mais qu'elles le sont dans des conditions qui ne sont pas identiques : les ouvrages féminins se bornent, me semble-t-il, à offrir une figuration imagée des registres d'expression ou des thèmes essentiels des poèmes, tandis que les réalisations divines entretiennent un dialogue

Le présent article constitue la version intégrale de l'étude dont un résumé figure dans les Actes du colloque de Grenoble «Homère virtuel » (20-22 novembre 2002) : Gaia 7 (2003), p. 135146.

2 De fait, une méditation sur les représentations artistiques chez Homère devrait s'inscrire dans des considérations plus générales sur tous les objets de spectacle ou de contemplation, et sur ce que F. Frontisi-Ducroux appelle la "thématique du spectacle ": La cithare d'Achille, Rome, 1986 (Biblioteca di Quaderni Urbinati di Cultura classica, 1), p. 62 sq. J'en avais amorcé l'examen dans D. AUBRIOT, «Représentation plastique et récit poétique : le Bouclier d'Achille ou les cuvres d'art en miroir" ", in M. CHEFDOR (éd.), De la palette à l'écritoire, vol. I, Nantes, 1997, p. 25-35. Il convient de rendre justice aux pages remarquables de P. Vivante, The Homeric Imagination. A Study of Homer's Poetic Perception of Reality, Bloomington / London, 1970, p. 25 $s q$., mais il est impossible de mentionner ici toutes les publications afférentes au sujet: on en trouvera l'essentiel dans les deux articles cités dans la note suivante, à quoi il conviendra d'ajouter S. DuBEL, "L'arme et la lyre : Remarques sur le sens du bouclier d'Achille dans l'Iliade ", Ktèma 20 (1995), p. 245-257; J. PIGEAUD, «Le Bouclier d'Achille. Essai sur l'interprétation du livre de Jean Boivin ", Ktèma 20 (1995), p. 259-272; S.G. BARBERO, "El Escudo de Aquiles y los limites de la Descripcion poética ", in Actas del III Simposio Nacional de Estudios Classicos, vol. II, Universidad Nacional de La Plata, 1997, p. 161-169.

${ }^{3}$ La présente réflexion part, pour les rassembler, d'éléments qui ont été développés séparément dans plusieurs études antérieures, principalement deux : D. AUBrot, "Imago lliadis. Le bouclier d'Achille et la poésie de l'lliade», Kennos 12 (1999), p. 9-56, et «Le détail et son double : fausse description, vraie narration; à propos d'Odyssée XIX, 225-33 ", in M. RICORD (éd.), Le parti du détail. Enjeux naratifs el descriplifs, Études romanesques 7 (2002), p. 139-153. En revanche il n'entre pas dans mon propos de reprendre ce qui a pu être dit concernant les métaphores artisanales du métier poétique. 
l'idée que les travaux d'Hélène et d'Andromaque ne sont pas des fioritures décoratives, mais constituent les emblèmes d'une complémentarité de signification essentielle au poème. Reste à scruter dans quelles conditions.

En effet cet examen trouverait rapidement ses limites si on ne le prolongeait par la considération de l'œuvre divine qu'est le bouclier d'Achille. Car par rapport à ces étoffes, œuvres féminines, la pièce d'art ouvrée « dans les

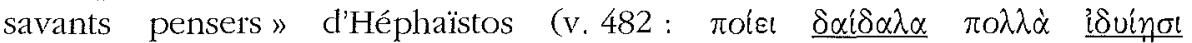
$\pi \varrho \alpha \pi i \delta \varepsilon \sigma \sigma l v)^{12}$ se présente à la fois en parallèle et en surenchère (II. XVIII, 478 sq.) : parallèle, en ce que l'hémistiche $\tau \varrho i \pi \lambda \alpha \kappa \alpha \mu \alpha \varrho \mu \alpha \varrho \varepsilon ́ \eta \nu$ qui ouvre la «description » du bouclier fait visiblement écho à celui qui introduisait (à la

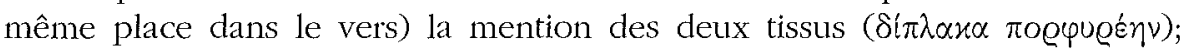
surenchère aussi, puisque le double est devenu triple, et que la pourpre royale a cédé la place à la splendeur adamantine et éternelle ${ }^{13}$ de l'œuvre d'un immortel.

\subsection{Le bouclier d'Achille (II. XVIII, 483-608)}

Sans vouloir revenir sur ce texte que j'ai étudié ici dans un numéro précédent $^{14}$, je rappellerai seulement que la prétendue « description » de ce bouclier offre la présentation de motifs successifs, à l'aide de verbes qui marquent le travail, la fabrication, la création, l'application, la décoration, et que la succession de ces verbes a surtout pour résultat d'attirer l'attention sur l'organisation discursive de cette énumération. Ce procédé (qui groupe les scènes selon leurs affinités) met en évidence une structure, suggère une conception abstraite, bien plus qu'il ne contribue à « décrire » quoi que ce soit de matériel.

\section{Les scènes reliées à la trame diégétique de l'Iliade (Il. XVIII, v. 490-540)}

Ainsi, la scène dite «du procès » (v. 497-508, où le débat roule sur l'alternative entre l'intransigeance et les accommodements) offre une congruence particulière à l'évolution d'Achille finalement contraint de renoncer à sa « colère », puis de rendre le corps d'Hector moyennant la rançon proposée par Priam ${ }^{15}$; ou bien encore la scène de la ville assiégée (v. 509 sq.) présente une transposition dans l'ordre général des menaces qui pèsent sur la ville de Troie. En un mot, ces scènes de la première série, qui sont placées sous le verbe $\pi$ oin $\sigma \varepsilon$ ( $v$. 490) me semblent reliées directement à la trame diégétique de l'Iliade. En conséquence, loin de considérer les épisodes narratifs contenus

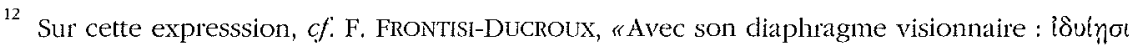
$\pi \varrho \alpha \pi i \delta \varepsilon \sigma \sigma \iota$, Iliade XVIII, 481. À propos du bouclier d'Achille n, REG 115 (2002), p. 463-484.

13 L'épithète $\mu \alpha \varrho \mu \alpha \varrho e ́ n\rangle$ ne sert par ailleurs dans l'lliade à qualifier que la mer divine (XIV, 273) et l'égide de Zeus (XVI, 594).

1. Cf. AubrioT, "Imago Iliadis" (supra, n. 3). Pour la justification des conclusions qui sont reprises ici comme un acquis, je me permets de renvoyer à cet article.

${ }^{15}$ Cf. D. Aubriot, Prière et conceptions religieuses en Grèce ancienne, Lyon, CMO, 1992, p. 204-207. Sur le rapport entre cette rétrocession finale et l'ambassade du chant IX, cf. D. AUBRIOT, «Remarques sur le chant $L X$ de l'lliade », BAGB, 1985 (3), p. 257-79 : p. 266-271. 
dans la " description » du bouclier d'Achille comme des faiblesses maladroites (où le poète, faute de savoir décrire une cuvre plastique immobile ${ }^{16}$, aurait dû se résigner à représenter ces scènes en mouvement, et même accompagnées de bruits, de sons articulés, et de musiques !), je propose d'y voir un remodelage délibéré des éléments de la narration principale (en l'occurrence la colère d'Achille, les dangers affrontés par les Troyens), remodelage conçu et formulé de manière à évincer toutes les anecdotes particulières et tous les noms propres ${ }^{17}$ : moyen puissant pour conférer à ce morceau un tour abstrait. Cela élargit à la fois l'horizon de la narration et le point de vue sur la vie humaine, conformément au recul qu'on peut attendre d'une ouvre divine, aperçue pour ainsi dire sub specie cetemitatis.

\section{La scène de la moisson, et la comparaison du chant XI (I1. XVIII, v. $550-60)^{18}$}

Quant aux trois vignettes agricoles annoncées par le verbe étí $\theta \varepsilon l$ (utilisé à trois reprises, aux vers 541, 550, 561) - qui font suite au long morceau regroupant sous un même $\pi$ oin $\sigma \varepsilon$ la ville en paix (v. 490-508), la ville en guerre (v. 509-534), et le sizain du «Trépas sanglant» (v. 535-540) -, elles sont situées exactement (grâce au même év dé : v. 490, 535, 541, 550, 561, 573 , etc.), sur le même plan que les vignettes reprenant la trame diégétique, mais elles nous replongent dans le monde des comparaisons ${ }^{19}$. Je ne reprendrai qu'un élément entre autres pour en faire foi : la deuxième de ces scènes (v. 550-560) représente «un domaine royal. Des ouvriers moissonnent... Des javelles tombent à terre les unes sur les autres, le long de la ligne

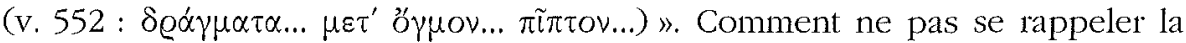
comparaison saisissante par laquelle commençait solennellement le récit des batailles du chant XI (v. 67 sq.) : "Ainsi que des moissonneurs qui, face les uns aux autres, vont, en suivant leur ligne... et les javelles tombent (v. 68-9:

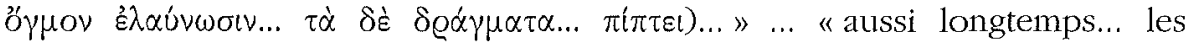
guerriers tombaient (v. $85: \pi \tilde{\imath} \tau \varepsilon \delta \varepsilon \dot{\varepsilon} \lambda \alpha \delta$ ) ) ? La scène figurée sur l'œuvre du dieu évoque trop la comparaison qui introduisait le récit des combats pour que ce soit un hasard ${ }^{20}$ : assurément à mon avis, elle en constitue un rappel

${ }^{16}$ Comme le laisse entendre C.H. WHITMAN, Homer and the Heroic Tradition, Cambridge, Mass., 1958, p. 205.

${ }^{17}$ Pas un nom propre dans tout le bouclier homis ceux de Dédale et d'Ariane ( $\left.v .592\right)-$

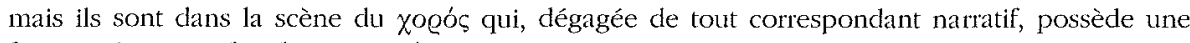
fonction à part : c/. infira, p. 53-54.

18 De fait la troisième de ces vignettes est un peu à part: $c f$. Aubriot, "Imago Iliadis" (supra, n. 3), p. 25-27.

19 Les vignettes pastorales quant à elles (v. 573-89) sont à nouveau mises sous "l'invocation", si l'on peut dire, du verbe $\pi$ olnoe - mais sans augment, cette fois : $c f$. L. BASSET, "L'augment et la distinction discours/récit dans l'lliade et l'Odyssée », in Étucles bomériques, TMO 17, Lyon, 1989, p. 9-16, car elles renvoient à l'allégorie animale des combattants (donc en quelque manière au récit des batailles).

${ }^{20}$ On ne peut invoquer l'argument du style formulaire, puisque ce sont des mots qui sont repris, et non des formules. 
conscient et signifiant, destiné à être perçu par l'auditeur ou le lecteur, quoique plusieurs milliers de vers les séparent.

\section{L'ouverture du chant XI, centrale pour le sens de l'Iliade}

Si l'on en doutait, on devrait s'aviser que d'autres indices désignent cette ouverture du chant XI comme un passage décisif pour l'architecture de l'Iliade, un passage à garder en mémoire pour qui tente d'embrasser les connexions internes du poème et donc d'en saisir le sens. En premier lieu, il est remarquable que cette comparaison des guerriers à des moissonneurs soit juste précédée par la seule autre mention circonstanciée d'un bouclier dans cette épopée : il s'agit de celui d'Agamemnon, qui est cette fois vraiment décrit, au milieu de la scène d'armement ${ }^{21}$ de l'Atride (v. 32-7). Le tableau offert par le bouclier du chef militaire, dépeint avec brièveté et précision, permet de mieux apprécier l'originalité de celui d'Achille ${ }^{22}$, et du même coup la diffétence qui sépare les deux héros : d'un côté le roi tout à son œuvre d'intimidation guerrière, de l'autre le demi-dieu mortel, presque indécis entre humanité et divinitée $e^{23}$ à la force surhumaine mais désabusé de tout. À cela il faut ajouter que ce début du chant XI est explicitement désigné comme essentiel à la signification de l'Iliade ${ }^{24}$ par le rappel formel qui y est fait de l'invo-

${ }^{21}$ Il. XI, 32-7: «Puis il prend son vaillant bouclier, qui le couvre tout entier; son beau bouclier ouvragé. On y voit sur les bords dix cercles de bronze, et, au centre, vingt bossettes d'airain, toutes blanches, sauf une, au milieu, de smalt sombre. Gorgone aussi s'y étale en couronne, visage dhorreur aux terribles regards, qu'entourent Terreur et Déroute. » Non seulement l'armement de l'Atride est décrit avec la solennité qui convient au personnage ( $\mathrm{v} .17$ sq.), mais toutes les pièces de l'armure sont énumérées, et quelques-unes sont décrites avec minutie. Il met d'abord ses jambières, sa cuirasse qui donne lieu à une description de dix vers, puis son épée enfermée dans un fourreau qui s'adapte à un porte-épée - toutes pièces dont l'origine, la décoration, ou la matière, nous sont indiquées avec un luxe impressionnant de détails descriptifs. Vient ensuite le bouclier (v. 32-7). Puis la scène d'armement se termine avec le baudrier, le casque à panache, et les deux piques (v. 38-45), tandis que la splendeur du souverain de Mycènes est saluée par un coup de tonnerre d'Athéna et d'Héra (un effet parallèle, mais amplifié à la dimension d'une véritable révolution cosmique, est développé au moment où Achille reparaît sur le champ de bataille : Il. XX, $41 \mathrm{sq}$., et c'est alors Zeus qui tonne. Sur ce passage, $c f$. D. AuBRiot, «Entre Héphaïstos et Poséidon : cataclysmes homériques », à paraître dans les Actes du colloque Connaissance el représentations des volcans dans l'Antiquité, qui s'est tenu à Clermont-Ferrand les 19-20 septembre 2002 sous la responsabilité d'Éric Fouton, p. 11-12 du manuscrit). Sur les scènes d'armement, cf. J.A. Armstrong, "The Arming Motif in the Iliad", AJPh 129, 4 (1958), p. 337-354.

22 Qu'on serait bien en peine de se représenter, et où dominent des spectacles apparemment paisibles.

${ }^{23}$ Comme j'ai tenté de le montrer dans D. AUBriot, "Humanité et divinité dans l'Iliade à travers le personnage d'Achille $n$, in M. WORONOFF, S. Follet, J. JouAnna (éds), Dieux; béros, et médecins grecs. Hommage à Fernand Robert, Presses Univ. Franc-Comtoises, 2001, p. 7-27.

${ }^{24}$ C'est d'autant plus frappant qu'on retrouve la même architecture dans l'Odjssée avec la reprise, vers le milieu du poème, du programme annoncé dans l'invocation. Cette constatation permet à la fois de dégager le même souci de composition dans chacune des deux épopées homériques, et de comprendre comment cette unité de plan gouverne non seulement chacune des deux cuvres, mais le rapport d'un poème à l'autre: $c f$. Aubrot, "Entre Héphaïstos et Poséidon " (supra, n. 21, p. 16 du manuscrit). 
cation initiale du poème, et par là, du " plan de Zeus ». Car si l'on pouvait, au

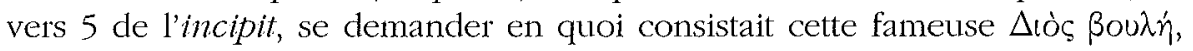
l'éclaircissement survient en cette séquence du chant XI, où nous apprenons que c'est Zeus en personne qui "s'apprêtait à jeter en pâture à Hadès bien des têtes fières » (Il. XI, 55-58)

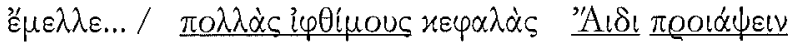

La reprise presque textuelle des vers 3 et 4 du chant I où figurait cette annonce (d'un point de vue restreint au Péléide : la colère d'Achille « jeta en pâture à Hadès bien des âmes fières / de héros »)

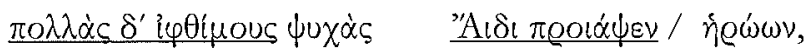
ne laisse aucun doute : le ressentiment d'Achille et ses conséquences doivent être intégrés au dessein de Zeus; et le fils de Thétis et de Pélée peut être regardé comme l'instrument du projet conçu par l'Olympien de mettre fin au monde des héros ${ }^{25}$. Toutes ces raisons convergent pour faire apercevoir le caractère central de l'ouverture du chant XI, et donc amener à saisir de quelle importance est la comparaison des guerriers à des moissonneurs pour le sens du poème : loin que l'fliade doive être comprise comme une exaltation des valeurs guerrières, elle en dégage au contraire subrepticement le caractère léthifère, sans cesser néanmoins de garder l'apparence d'une épopée traditionnelle chantant les prouesses héroïques. On ne saurait donc surestimer le poids de la mise en regard du chant XI en son début (quand reprennent les grandes batailles après la longue interruption de l'Ambassade et de la Dolonie) et des scènes agricoles sur le bouclier ${ }^{26}$ : elle atteste que les vignettes figurant sur l'œuvre d'Héphaïstos ne répondent à aucune prétention descriptive, mais constituent une pièce de la narration - en même temps qu'elles en renversent le sens apparent.

\section{L'ouvre du dieu et sa correspondance avec celle du poète}

Ainsi, quand le poète affecte de céder la place au dieu dans la création d'une cuvre d'art (qui serait plastique et non plus aédique), c'est une illusion : cette «description»-qui ne comporte ni dessus ni dessous ni droite ni gauche, qui montre des scènes dynamiques (et sonores !), qui dépeint des changements chromatiques (figurant l'or en train de noircir : v. 548-9) - n'en est pas une. Comme les ouvrages des femmes, l'œuvre du dieu doit être considérée dans son rapport à celle du poète. Il y a cependant une différence importante: si nous trouvons sur le bouclier réalisé par Héphaïstos, à travers les scènes des villes et celles des champs, la même complémentarité qui nous

\footnotetext{
25 Je suis obligée ici de passer vite sur ce point pourtant essentiel, mais j'ai pour ma part essayé de montrer (dans l'art. cité supra, n. 21) que la $\Delta$ ò̀ ßoùń incluait lá fin des héros, en examinant les rôles d'Héphaïstos et de Poséidon à travers les poèmes homériques. Il m'a été

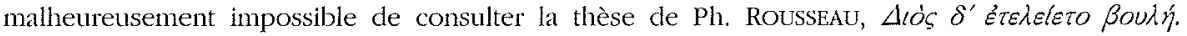
Destin des héros et dessein de Zetus dans l'intrigue de lTliade, Lille, 1995, qui sans doute aurait apporté beaucoup à cette démonstration.

26 On peut naturellement se livrer à des remarques comparables avec les autres tableaux représentés sur le bouclier.
} 
avait semblé marquer les deux étoffes tissées respectivement par Hélène et par Andromaque (d'une part le monde des combats et de la mort violente, de l'autre le monde de la nature et de la vie - mais aperçu sur le mode tragique de sa fragilité), si donc le bouclier d'Achille rassemble les deux registres qui étaient séparés sur les pièces tissées par les femmes, ce n'est pas seulement pour en opérer une synthèse, mais pour en modifier le sens. Car la réunion côte à côte sur le bouclier, cette fois au même niveau ${ }^{27}$, des thèmes qui étaient disjoints sur les deux étoffes et (au sens propre, syntaxique) "subordonnés" l'un à l'autre dans la narration poétique, fait sentir que l'équilibre est totalement modifié. Sur l'ouvrage de l'artiste divin, il ne s'agit plus d'accorder le premier plan aux prouesses hérö̈ques, de montrer des héros visant à la gloire d'être «semblables aux dieux», soucieux de pérenniser leur mémoire grâce à de grands exploits qui seront chantés par la poésie ${ }^{28}$, ni de laisser dans la pénombre de propositions dépendantes les scènes de vie cantonnées dans les comparaisons, ou de consacrer un développement moindre à l'ouvrage d'Andromaque et aux fleurs qui le constellent. L'œuvre d'Héphaïstos au contraire dégage face à face et sur un même plan les mirages fatals de la gloire qui s'obtient au combat, et tout ce qui fait la sève de la vie. Toute cette imitation, sur le bouclier, du monde précaire des "mortels vivants »" est présentée en opposition à un monde des dieux qui est lumineux, majestueux, radicalement supérieur et parfaitement solidaire (et non pas divisé en clans, voire mêlé aux humains, comme dans le poème) ${ }^{3 n}$. C'est-à-dire que l'art divin remet à sa place la vaine espérance dont les hommes se grisent, de jouir d'une sorte de familiarité avec les Immortels. Or ce qui contribue le plus à signaler et à soutenir cette modification de perspective, c'est le traitement que reçoivent sur le bouclier les procédés poétiques : ce ne sont plus des récits de combats à mort qui s'appuient sur des comparaisons intervenant, en subordination, comme des intermèdes; ce sont, en son milieu, d'abord des scènes géorgiques qui convoquent (sous leur apparence idyllique) le souvenir des passages de bataille les plus sanglants, puis un peu plus loin des vignettes bucoliques qui consomment la transposition des cruautés humaines dans un monde animal maculé de la bestialité la plus farouche ${ }^{31}$.

Ainsi, toutes les scènes du bouclier, celles des villes (qui évoquent de si près les péripéties sous Ilion), comme celles des champs (qui reprennent la thématique des comparaisons), sont placées strictement à égalité, selon une

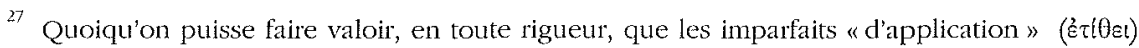

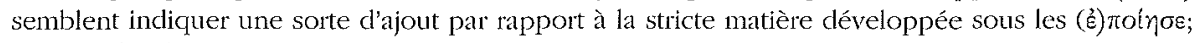
mais les év $\delta$ é viennent contrebalancer cette remarque en plaçant tout au même niveau.

${ }^{28}$ Cf. D. Bouviar, Le sceptre et la lyre. L'Iliade ou les héros de la mémoire, Grenoble, 2002.

29. XVII, 539 : לwoi $\beta$ gotol.

30 Il. XVIII, 516-519. En particulier Arès et Athéna agissent de concert.

31 Il s'agit bien encore (v. 573-586) d'utilisation et de reprise de comparaisons, mais dans une autre perspective que pour les vignettes agricoles, cette fois avec une imagerie symbolique propre à laisser transparaître des protagonistes humains particuliers derrière les bêtes ( $c f$. Aubior, "Imago Iliadis" [supra, n. 3], p. 29-31, pour montrer que les deux lions qui « hument les entrailles et le sang noir » du taureau sont les deux Atrides de XI, 176, et de XVII, 64). 
technique de récit morcelé qui sertit chaque scène, indépendamment des autres, hors du temps et de l'espace ${ }^{32}$, dans un total anonymat, in abstracto. Il en résulte une impression globale de détachement par rapport aux contingences accidentelles, qui relègue la vie et la mort dans le même lointain. Aussi observe-t-on que, contrairement à d'autres objets artistement façonnés qui sont dits «merveille à voir », le bouclier d'Achille est simplement (et bien plus largement) acclamé comme "merveille » ( $\theta \alpha \tilde{u} \mu \alpha$ : v. 549 et non $\theta \alpha \tilde{u} \mu \alpha$

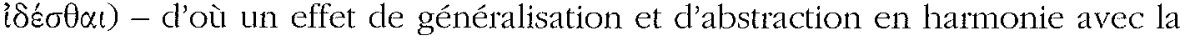
distance dont je parlais à l'instant. Tout concourt donc à faire sentir que sur le chef-d'œuvre divin, les points de vue sur l'énergie vitale et l'anéantissement reçoivent un éclairage totalement transposé, où les valeurs sont déplacées, voire inversées, comme il sied quand une perspective divine se substitue à la perception humaine, mais aussi quand l'idéologie héroïque s'efface derrière les réalités de la vie quotidienne.

\section{Le rôle de l'art, et les relations entre bommes et dieux}

Cependant, il ne faut pas perdre de vue que l'ouvrage d'Héphaïstos luimême n'est rien d'autre qu'une création du poète : si ce bouclier est bien " indescriptible » ${ }^{34}$, c'est que sa seule existence, en fin de compte, est poétique. Aussi bien la pseudo-description constituée par ce morceau est-elle couronnée par la scène du Xo@ós (v. 590-606), que tout désigne à l'attention ${ }^{35}$, et qui elle-mêtne culmine dans la représentation d'un aède autour duquel tout tourne; en sorte que par un jeu de miroirs unique en sa double réverbération, le poète (de l'fliade) montre un dieu confectionnant une ouvre (le bouclier) qui elle-même, au pinacle de sa scène la plus exceptionnelle, place un aède.

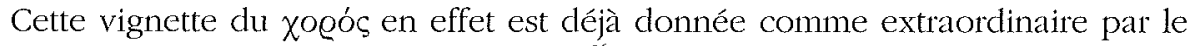
verbe qui sert à la présenter, $\pi$ oi $\chi \lambda \lambda \varepsilon^{36}$ : il suffit à indiquer l'intention artistique nettement recherchée, et souligne d'emblée qu'il faut voir dans ce passage le fleuron de l'œuvre divine. Cette scène par ailleurs se distingue des autres vignettes en ce qu'elle ne possède aucun correspondant narratif (alors que les autres font écho, nous l'avons vu, soit au récit des événements qui se déroulent dans la plaine troyenne, soit à la matière des comparaisons). En cela, tout en ayant l'air de prendre la suite des vignettes précédentes (en

${ }^{32}$ Sur quoi $c f$. D. AuBriot, "Non-paysage, para-paysage, et anti-paysage dans l'fliade », in M. Perrin (éd.), L'idée de Pajsage, Greifswald, Reineke Verlag, 1997, p. 5-29 : p. 8-15.

33 Cf. R.A. PrIET, Thatma Idestbai: The Pbenomenology of Sighl and Appearance in Archaic Greek, The Florida State University Press, 1989; C. HunZINGER, "Le plaisir esthétique dans l'épopée archaïque », $B A G B, 1994$ (1), p. 4-30: p. 7.

34 Cf. F. LÉTOUBLon, "L'indescriptible bouclier", in Euphrosyne. Studies in Ancient Epic and its Legacy. In Honor of Dimitris N. Maronitis, Stuttgart, 1999, p. 211-20.

${ }^{35}$ Cf. AUBriot, «Imago Iliadis » (supia, n. 3), p. 39-52.

${ }^{36}$ Non seulement il n'a pas encore été employé dans cette description, mais de surcrôt c'est un bapax dans l'Ihade. Sur les mots de cette racine et leur aptitude à s'employer pour les travaux textiles (surtout les travaux d'aiguille, mais aussi le tissage) autant que pour le travail du métal, $c f$.

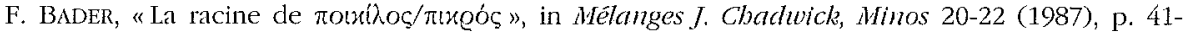
60 : p. 51-52. 
commençant toujours par le même év $\delta \dot{\varepsilon}$ ), elle se situe sur un autre plan, et constitue une sorte de transition avec les vers d'ouverture et de clôture du bouclier, qui dessinent une mise en place proprement cosmique de ce «microcosme »" figuré par le dieu. Nombreux sont les autres détails qui viennent encore s'accumuler pour confirmer le statut à part de cette scène du

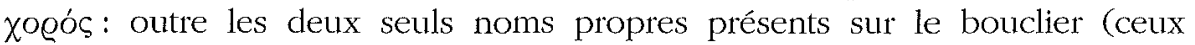
d'Ariane et de Dédale ${ }^{38}$ ), on y relève la concentration de toute une série de savoirs subtils, réunis pour rehausser des cérémonies cultuelles, et par là propres à suggérer l'importance de l'art dans les relations entre hommes et dieux ${ }^{39}$. Ce n'est certainement pas un hasard si leur sommet réside justement dans la présentation de cet aède autour de qui tout s'ordonne, en un vers (604) qu'à mon avis il s'impose de retenir ${ }^{40}$. En effet par la place qu'occupe la mention: "Et parmi eux chantait un aède divin, s'accompagnant de la cithare ", cette vignette nous offre le spectacle unique et infiniment lourd de sens d'un aède, élément suprême, fixe et central (entouré qu'il est d'acrobates faisant la roue pour «préluder au chant» et d'auditeurs qui font cercle autour

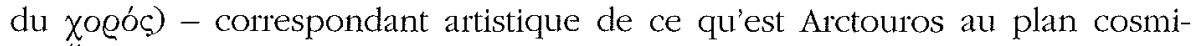
$q^{\prime \prime} e^{41}$. C'est en tout cas ce que suggère presque explicitement la mention

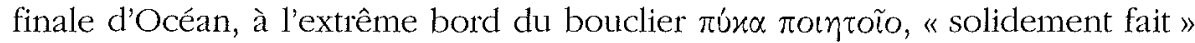
- ou "solidement pensé »-, expression dont on ne sait s'il faut l'appliquer à la réalisation matérielle du bouclier, à sa conception générale par « l'illustre Boiteux »" à sa «description» poétique, ou aux trois à la fois. Mais cette ambiguité elle-même, significative sans aucun doute, invite à ne pas manquer les implications du jeu spéculaire grâce auquel l'aède figure sur l'œuvre divine dans la même situation que le dieu dans l'œuvre poétique. Ainsi se trouve mise en lumière la place éminente de l'exécution artistique, et en particulier de la création poétique, dans des relations entre hommes et dieux où la communication rituelle vient remplacer l'intimité filiale (au propre ou au figuré) qui unissait les héros aux Immortels.

Il semble donc bien qu'on puisse l'affirmer : les représentations artistiques remplissent dans l'Iliade une fonction qu'on ne saurait appréhender sans la rapporter au corps même du poème, dans des conditions qui invitent à

37 En effet (même si cette interprétation me semble insuffisante), le bouclier est bien «Imago mundi $\gg$.

${ }^{38}$ Que le poète signale explicitement comme un modèle pour le dieu : XVIII, 591-592.

39 C'est un point qui mérite d'être mis en lumière. C. Calame le suggère dans plusieurs de ses publications: cf: entre autres «L'hymne homérique à Déméter comme offrande : regard rétrospectif sur quelques catégories de l'anthropologie de la religion grecque ", Kernos 10 (1997), p. 111-133; ou encore «Variations énonciatives, relations avec les dieux et fonctions poétiques dans les Hymnes bomériques ", MHI 52 (1995), p. 2-19, et "Rythme, voix et mémoire de l'écriture en Grèce classique », in R. PRETAGOSTINI (éd.), Tradizione e imovazione nelle cultura greca da Omero all'etã ellenistica. Scrilti in Onore di Bruno Gentili, Rome, 1993, p. 785-799); et il en est question dans l'art. cité infía, n. 48.

41) Ce vers est absent des manuscrits mais il nous est transmis par Athénée (181d).

4 Cf. AUBRIOT, "Imago Iliadis " (supra, n. 3), n. 170.

12 Sur quoi $c f$. Aubriot, «Entre Héphaïstos et Poséidon " (stupra, n. 21). 
regarder différemment l'œuvre divine et les réalisations féminines. Les ouvrages textiles des femmes décomposent la matière poétique en ses éléments: les combats du récit pour ceux d'Hélène, la végétation vivante ${ }^{43}$ des comparaisons pour ceux d'Andromaque. Le bouclier forgé par Héphaïstos en revanche présente sur le même plan une transfiguration de ces deux matériaux poétiques : si les travaux des femmes les séparaient, si le poète les entrelaçait en les subordonnant l'un à l'autre (grâce, précisément, à des subordonnées comparatives), le dieu quant à lui les place à même distance - égalité de traitement rendue manifeste par les év $\delta \varepsilon ́$ successifs. Par cette modification du rapport entre les batailles et les réalités de la vie, la suprématie des aventures guerrières est rompue: ainsi est suggérée une relégation définitive des exploits héroïques - qui semble aller de pair avec le "plan de Zeus »" d'éliminer les héros. Sans doute n'est-il pas indifférent que soit affirmée en même temps, à travers la dernière vignette, la place essentielle de l'art (et en particulier de l'art du poète) dans le monde ainsi réorganisé, où les relations avec les dieux banniront la familiarité hérö̈que pour passer désormais par le culte. Au sein de ce nouvel ordre, le seul truchement qui reste possible entre hommes et dieux est l'artiste : là où l'héroïsme échoue (aussi bien celui que

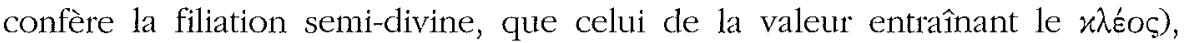

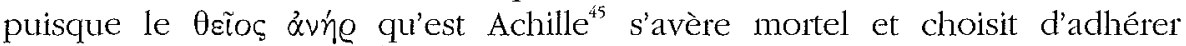
pleinetment à l'humanitét ${ }^{46}$, le $\theta \varepsilon \tilde{i} o \varsigma_{\alpha} \alpha o t \delta o ́ s$, lui (XVIII, 604), a pouvoir d'obtenir une forme d'immortalité grâce à son ouvre ${ }^{47}$ et au plaisir qu'il procure à travers elle : la transsubstantiation de la glaise en or trouve son équivalent dans la sublimation des souffrances et de la mort en beauté artistique. La jouissance du plaisir ainsi produit, partagée qu'elle est par hommes et dieux ${ }^{48}$, est un instrument privilégié de la communication qui s'établit entre eux ${ }^{49}$. Il convenait que ce fût un dieu qui, en proposant sur son cuvre une image réfractée de l'Iliade, en transcendât le sens, car lui seul avait l'autorité voulue pour auréoler ainsi l'art du poète.

43 Sur le rapport, constant dans la pensée grecque, entre l'homme et la végétation, $c$. Aubiziot, «L'homme-végétal : métamorphose, symbole, métaphore », in E. Delruelle, V. PiRfnNeDelforge (éds), Kìnoı. De la religion à la pbilosopbie. Mélanges offerts à André Motte, Liège, 2001 (Kemos, suppl. 11), p. 51-62.

44 sipia, n. 25.

45 Il. XVI, 798 : c'est bien le seul endroit où l'on trouve (au génitif) cette alliance frappante,

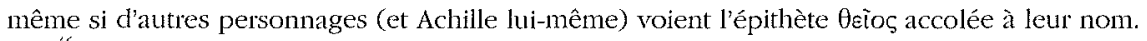

4 4' Cf. AuBriot, «Humanité et divinité » (supra, n. 23).

47 Sans doute cette ceuvre contient-elle encore une exaltation du $x \lambda \lambda^{2} o \varsigma$ né des exploits (sur la mémoire, cf. D. BOUVIER, "La mémoire et la mort dans la religion homérique ", in Kernos 12 [1999], p. 57-71). Mais cette exaltation est largement tempérée par le fait que ces exploits sont si durement entachés de cruauté.

4s $C f$. D. Aubriot, "Autour de quelques fêtes épiques et lyriques: Homère et Pindare ", in A. MotTe, Ch.M. Ternes (éds), Dieux; Fêtes, Sacré dans la Grèce et la Rome antiques. Actes dut colloque de Luxembourg, les 24-26 octobre 1999, Brepols, 2002, p. 53-80.

49 Puisque toute cérémonie comporte des chants (cf. les art. cités supra, n. 39). 
On ne s'étonnera pas que l'Odyssée, qui se situe résolument sur le plan humain, permette des constatations légèrement différentes; mais on estimera d'autant plus précieux de les trouver cohérentes avec le statut des représentations artistiques chez Homère aperçu jusqu'ici.

\section{L'Odyssée}

Ce poème conduit à la fois à retrouver et à préciser ce que l'Iliade nous avait permis de dégager. Pour m'expliquer, je commencerai par m'intéresser à un détail descriptif qui, à ma connaissance, n'a guère retenu l'attention jusqu'ic ${ }^{50}$. Il s'agit de la parure qui ornait le vêtement d'Ulysse au moment de son départ.

\subsection{La "broche" (ou broderie) et les chiens : le système symbolique}

\section{L'ornement du manteau d'Ulysse et les détails apparemment superflus}

Le passage se situe dans lá grand salle du palais d'Ithaque, au moment où le maître (rentré chez lui sous le déguisement d'un mendiant) prétend, pour obtenir confiance et sympathie, avoir accueilli Ulysse par le passé. Il est alors sommé par la reine de fournir des preuves de ses dires. C'est donc Ulysse en personne qui, sous une identité d'emprunt, dépeint pour Pénélope (qui ne l'a pas encore reconnu) la vêture du voyageur qu'il déclare avoir vu : «Le divin

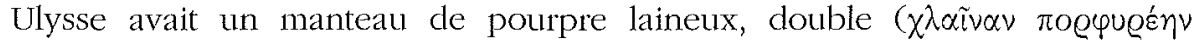
oủ $\lambda \eta \nu . . . \delta(\pi \lambda \tilde{\eta} \nu)$; sur celui-ci se trouvait une agrafe en or à deux tuyaux

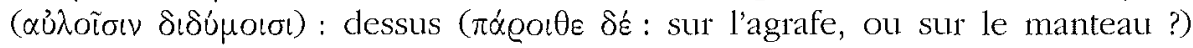
était un ouvrage d'art ( $\delta \alpha i \delta \alpha \lambda o v)$; dans ses pattes de devant, un chien tenait un jeune cerf tacheté, qui se débattait, tandis que le chien le regardait

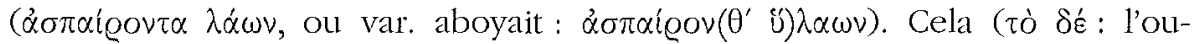
vrage, la scène ?) tous étaient à l'admirer; car les animaux étaient en or: le chien regardait le faon en l'étranglant, tandis que le faon, plein du désir de

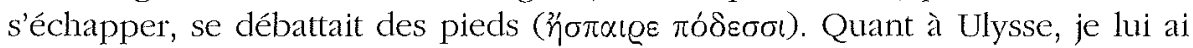
remarqué sur la peau une tunique... (fine...) brillante comme le soleil; (nombreuses, oui, étaient les femmes qui la contemplaient...) »".

Cette «description» appelle plusieurs remarques. Premièrement, il ne semble pas douteux que le "manteau » (car cette fois c'en est bien un, explicitement : $\chi \lambda \alpha \tilde{\nu}(\alpha \nu)$ nous ramène aux ouvrages d'Hélène et d'Andromaque, en ce que lui aussi est dit « de pourpre » et «double». Néanmoins l'expression

\footnotetext{
50 Mais dont l'examen constitue la base de l'article «Le détail et son double » (cf. supra, n. 3).

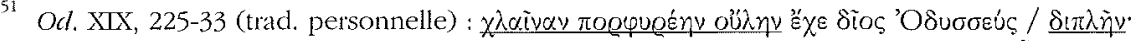

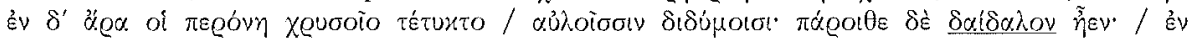

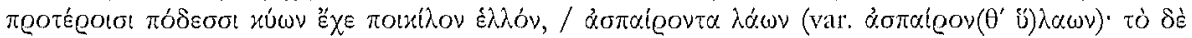

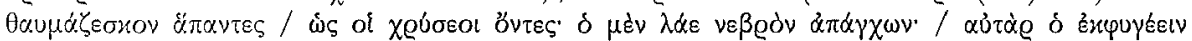

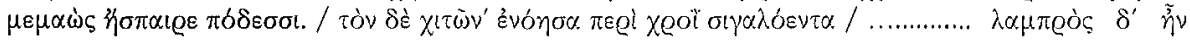
$\hat{\eta}^{\prime} \hat{\prime} \lambda \operatorname{los}{ }^{\omega} \mathrm{c}^{\circ}$
} 


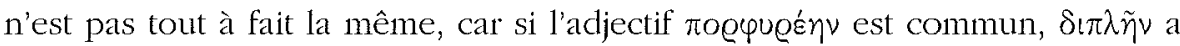
remplacé $\delta i \pi \lambda \alpha \kappa \alpha$. Comme de surcroît ce manteau est «laineux» ( $\chi \lambda \alpha \tilde{i} v \alpha \nu \ldots$ oú $\left.\lambda \eta \nu_{\nu}\right)$, on voit qu'on est passé de l'abstrait au concret ${ }^{52}$. Deuxièmement, peutêtre est-il symptomatique qu'on ne puisse savoir au juste ici (car $\pi \dot{\alpha} \varrho o ı \theta \varepsilon \delta \varepsilon$ est vague) si «l'ouvrage d'art » décrit ( $\delta \alpha i \delta \alpha \lambda o v)$ a été travaillé par un orfèvre, c'est-à-dire si c'est une broche (comme les bijoux fabriqués par Héphaïstos pour Thétis et Eurynome en Il. XVIII, 401) ou si c'est une broderie appliquée sur le manteau au moyen de fils d'or - ce qui nous ramènerait au genre de travaux réalisés par Hélène et Andromaque : il faudrait alors adjoindre Pénélope aux deux autres femmes dont les ouviages textiles offraient une transposition plastique de la matière poétique. En tout cas, cette incertitude provisoire (sans doute suscitée à dessein avant d'être levée un peu plus tard) nous laisse, le temps de cette description, aux prises avec les termes de l'alternative que nous soulignions tout au début, entre représentations ciselées dans le métal, et broderies ou tissages figurés sur ou dans l'étoffe ${ }^{53}$. Troisièmement, la « description » semble curieusement menée, puisqu'elle comporte diverses bizarreries, précisions apparemment superfétatoires, voire redites formelles - toutes anomalies concentrées dans les deux vers 230-31 (indiqués ici en caractères gras), qu'au reste V. Bérard propose d'évincer du texte. En effet, dans ce distique, le lecteur se trouve confronté aux surprises suivantes. Tout d'abord l'indication "le chien regardait le faon en l'étranglant " (ó $\mu \dot{\varepsilon} v$ $\lambda \alpha \dot{\alpha} \varepsilon \varepsilon \beta \varrho o ̀ v ~ \alpha \dot{\alpha} \alpha \gamma \gamma(\omega v)$ a quelque chose d'étrange, car on voit mal comment un chien pourrait regarder une bête qu'il tient à la gorge. De plus, ces deux vers insistent sur des circonstances impossibles à figurer plastiquement: une agitation dynamique ( se débattaient»), et plus encore l'intention qui la commande (« lésirant s'échapper»). Encore serait-on en droit d'arguer que cela ne surprend pas dans une description homérique, et que le bouclier d'Achille suscitait le même genre d'étonnement. Néanmoins on pourrait estimer que la mention des affres de l'animal (« désirant s'échapper») est

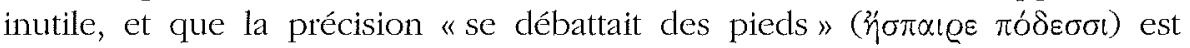

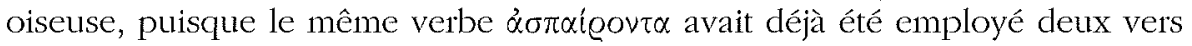
avant et suffisait à décrire le mouvement convulsif. Tout se passe en fait comme si ces adjonctions étaient une vaine réduplication de l'indication concise qui précècle : "Dans ses pattes de devant un chien tenait un jeune cerf tacheté, qui se débattait tandis que le chien le regardait », comme si par conséquent ce distique était redondant et en tout cas superflu. Il parât l'être

\footnotetext{
${ }^{52}$ Ce qui est en parfaite conformité avec la différence des préoccupations de l'Iliade et de l'Oclyssée.

${ }^{53}$ Sans doute une consiclération attentive des marques d'admiration accordées à la vêture d'ulysse fait-elle, dès cette scène, pencher en faveur de la première solution, selon laquelle il s'agit d'une broche, et non d'une broderie. En effet «tous " admirent "l'ouvrage d'art " (plutôt que la scène qui y est représentée: v. 229, tò $\delta \dot{c}$ ), tandis que les vêtements proprement dits provoquent l'ébahissement des femmes seules (v. 235). Au reste, l'incertitude est levée une trentaine de vers plus loin, quand Pénélope en personne affirme (v. 255-7) que la parure d'Ulysse

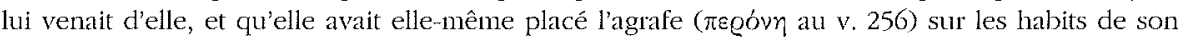
époux. L'ambiguité qui a été laissée un moment en suspens n'en est pas moins révélatrice.
} 


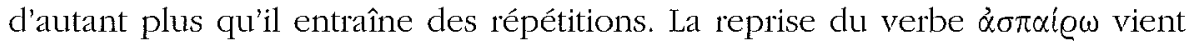
d'être notée. Par ailleurs, s'il faut bien lire au vers 229 «tandis que le chien le regardait » ( $\lambda \dot{\alpha} \varepsilon$ et non pas «aboyait»: un scholiaste rapporte que les grammairiens alexandrins en discutaient déjà), cela porte à deux $(\lambda \dot{\alpha} \varepsilon / \lambda \dot{\alpha} \omega \nu$, verbe inusité par ailleurs, et $\dot{\alpha} \sigma \pi \alpha \hat{i} \varrho \circ \tau \tau \alpha / \not ̈ \sigma \pi \alpha \varrho €)$ les reprises textuelles de ces trois vers - ce qui est faire de la répétition un usage curieusement insistant, même en tenant compte des habitudes stylistiques de l'épopée. En somme, la présence de ces deux vers litigieux a de quoi gêner, si l'on ne discerne aucun motif à leur incorporation en cet endroit. En effet, les seules nouveautés qui y soient introduites se résument à trois détails infimes en apparence : «en l'étranglant », « avec les pieds », et " désirant s'échapper" ».

\section{Les rapports entre les chants XIX et XXII de l'Odyssée}

Or ce sont justement ces détails à première vue inutiles ici qui alertent sur un possible rapport entre ces vers prétendument descriptifs du chant XIX, et un passage narratif ultérieur de l'Odyssée : il prend place pendant la séquence décisive où Ulysse exécute le châtiment de ses adversaires. Au chant XXII, dans un morceau savamment orchestré (v. 417-73), le poète raconte le supplice des servantes infidèles; cette peine, administrée par Télémaque, fait partie intégrante de la vengeance de son père. Ulysse vient de transpercer un à un les prétendants, de punir leurs complices les plus compromis, et de forcer les servantes qui avaient "collaboré » à emporter au dehors les cadavres de leurs amants puis à rentrer nettoyer la salle. Le père et le fils font ensuite ressottir les femmes et acculent leur troupe en un coin de la cour où "en aucune manière, il n'était possible de fuir" (v. 460). Là Télémaque, pour leur infliger la mort la plus infamante, décide de leur faire subir la pendaison en les accrochant toutes à un câble pour les étrangler comme grives ou

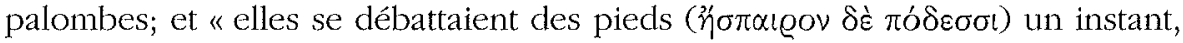
mais très bref» ( $v .473)$. En cette expression réside, il faut en convenir, l'unique similitude textuelle par où le chant XXII rappelle le chant XIX. Mais

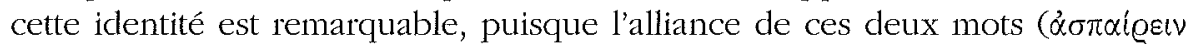
$\pi \delta ́ \delta \varepsilon \sigma \sigma l)$ ne se trouve dans l'Odyssée nulle part ailleurs. De plus, le contexte est le même (impossibilité de s'échapper, strangulation), quoiqu'on n'ait pas affaire à une récurrence formelle stricte pour ces deux termes. En sorte qu'on est bien tenté de considérer le récit du trépas de ces traîtresses, si essentiel à l'épisode fondamental de la vengeance qu'il vient couronner, comme une amplification de la mort du faon - détail qui, considéré à sa place, semblait n'être qu'une décoration facultative à sujet anecdotique. Cependant, pour que la confrontation des deux " descriptions » - celle de l'œuvre plastique (XIX) et celle du récit central (XXII) - présente un intérêt véritable, il faudrait que cette similitude de vocabulaire et de situation soit soutenue par d'autres indices. Or c'est bel et bien le cas. 


\section{Le symbolisme des faons}

Si l'on peut suggérer de voir dans la scène figurant sur l'agrafe une transposition anticipée de la vengeance d'Ulysse ${ }^{51}$, c'est pour une grande part en raison de l'imagerie animale qui y est employée. En effet, aussi bien le faon que le chien sont pourvus dans l'Odyssée d'une valeur symbolique certaine. Le nom veß@ó $\varsigma^{55}$ se retrouve en ce poème seulement dans deux autres passages (qui de fait sont la répétition textuelle l'un de l'autre), dans des conditions où le rapport à la vengeance d'Ulysse est indiqué avec netteté. La première fois (vers le début du poème) Ménélas, recevant Télémaque, l'a interrogé pour connaitre le but de sa visite et le jeune homme lui a, pour réponse, décrit l'état du palais familial en proie aux exactions des prétendants. Sur quoi l'Atride commente ainsi la situation (IV, 333 sq.) : " Ah, misère ! Voilà donc comme ils veulent se coucher au lit d'un brave, eux qui sont des lâches! Ainsi une biche a déposé dans le repaire (le lit: $\xi u \lambda o ́ \chi \omega)$ d'un lion robuste ses faons (veßgoúc) nouveaul-nés... et voici que le lion, rentrant dans sa tanière (sa couche : eủvñv), envoie aux... faons un destin misérable. Ainsi Ulysse leur enverra un destin misérable. Si seulement... il pouvait revenir... tel qu'il était jadis... la vie des prétendants serait brève et leurs noces amères! » Au chant XVII ces paroles sont reprises mot pour mot $^{56}$ par Télémaque qui, de retour à Ithaque, raconte son voyage à sa mère et lui répète exactement la réplique de Ménélas (v. 124 sq.). Or ce qui est ainsi rappelé à peu de distance de notre épisode (si bien que le souvenir en est encore tout frais au chant XIX) est une comparaison où les prétendants sont clairement désignés comme des faons immatures et inconscients du danger. Ce procédé aussi bien que sa répétition aident à comprendre le symbolisme de la représentation figurant sur la broche. Cependant, dira-t-on, si l'assimilation des faons (veß@oí) aux prétendants et à leurs séides ne peut donc faire aucun doute, l'animal qui les extermine a changé, lui, entre la comparaison et la description: Ulysse, qui dans la bouche de Ménélas était assimilé à un lion, est devenu sur l'agrafe un chien.

\section{La valeur symbolique du chien}

Mais le chien est un animal dont l'image touche de près au roi d'Ithaque. Il n'est pas besoin en effet de rappeler l'extraordinaire complicité qui unit Argos à son maître, ni le ventre « chien » d'Ulysse qui crie famine (VII, v. 216), ou encore son coeur " aboyant » d'indignation - précisément devant l'inconduite des servantes aux bras des prétendants (XX, v, 13-18, i.e. à peine quatre cents

54 Cf. Aubrior, «Le détail et son double » (cf. supra, n. 3), p. 145. On y trouvera évidemment d'autres raisons de nature à étayer l'interprétation rappelée ici.

55 C'est celui qui figure en $O d$. XIX, 230, dans le distique qui nous occupe, tandis qu'au

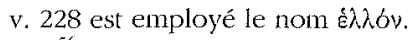

56 L'insistance marquée par la reprise textuelle des mêmes vers a retenu l'attention cle N. FELSON-Rubin, Regarding Penelope. From Character to Poetics, Princeton UP, 1994, p. 346. 
ver's après le passage sur l'agrafe) : tout cela est bien connu ${ }^{57}$. De plus Beck a démonté admirablement le mécanisme concerté selon lequel, tout au long de l'Odyssée, les trois thèmes du chien, de la maison, et du maître sont entrelacés et traités (pas seulement à propos d'Ulysse) de manière à désigner la même réalité, comme si maison et chien représentaient le maître aussi sûrement que le fait sa propre personne $e^{58}$. Il ne faut donc pas douter qu'on ait affaire à un système symbolique délibérément construit et cohérent, dont cette représentation vient ajouter une marque supplémentaire : le chien étranglant le faon qui se débat en vain est une métaphore prémonitoire non seulement d'Ulysse triomphant, mais aussi de sa "maison» (au sens large, en englobant la maisonnée des fidèles) coupant le souffle de la vie aux prétendants et à leurs complices $^{59}$. Il n'est pas jusqu'au changement d'animal survenant entre la comparaison faite par Ménélas et la description de la broche qui ne soit explicable et signifiant. L'Atride, tributaire de l'idéologie héroïque et qui a dans l'esprit un Ulysse guerrier, le compare comme on peut s'y attendre à un lion (figure ordinaire du héros d'exception, valeureux aux armes) ${ }^{60}$. En revanche sur le bijou agrafé par Pénélope (l'épouse), c'est du maître de la maison qu'il s'agit sous l'image d'un chien. Aussi Ulysse et Télémaque agissant de concert peuvent-ils être tous deux subsumés sous cette métaphore. Par conséquent, aussi bien les faons massacrés dans la couche du lion que le chien étouffant le jeune cervidé sont à considérer (sans témérité, je crois), comme une allégorie du retour et de la vengeance du maître.

Il me semble donc que cette sorte de blason figurant sur le manteau ${ }^{61}$ d'Ulysse à son départ n'est pas moins qu'une anticipation (fixée en un quasiinstantané) du triomphe final de la dynastie légitime sur ses adversaires. C'està-dire qu'une fois encore une représentation artistique sert d'équivalent trans-

57 Sur Argos on peut citer A. MOREAU, "D'Homère à Giono : l'animal fidèle ", in Actes du XXW ${ }^{r}$ Congrès de l'APLAES, Montpellicr, 1997, p. 61-71. Sur le ventre «chien », cf. F. BADFR, "L'homme et la bête, le cuit et le cru. Le gaster d'Ulysse et l'orgie dionysiaque de Polyphème ", Diotima 30 (2002), p. 155-168. Sur le coeur "aboyant», cf. J. DE ROMLLY, "Patience, mon cour ! „. L'essor de la psychologie dans la litterature grecque classique, Paris, 1984, p. 41.

${ }^{58}$ Cf. W. Bгск, "Dogs, Dwellings and Masters: Ensemble and Symbols in the Odyssey", Hermes 119 (1991), p. 158-167 (mais s'il clégage plusieurs échos entre le passage de la broche et le reste de l'Odyssée, il n'a pas vu le rapport au châtiment des servantes).

59 C'est bien ainsi en effet que se présente la mort en ce passage du chant XXII : noud coulant qui étrangle (v. 33, 41), souffle qui s'altère (v. 87), et surtout flot de sang obstruant les narines, avec justement le mot aù hó (v. 18) qui se retrouve en notre chant XIX au v. 227 - dans des conditions quelque peu énigmatiques, si l'on n'aperçoit pas que son emploi est commandé précisément par l'intention d'aboutir à ce rapprochement (ç. AUbriot, "Le détail et son double " [cf. supra, n. 3], p. 150).

6) Cf. A. SCHNAPP-GourbeILon, Lions, héros el masques. Les représentations de l'animal chez Homère, Paris, 1981, et surtout M. CLARKE, «Between Lions and Men : Images of the Hero in the Iliad », GRBS 36 (1995), p. 137-159.

${ }^{6} \mathrm{Je}$ ne commente pas ici l'ambiguitté selon laquelle $\chi$ hoivo peut désigner aussi bien un manteau que la couverture d'un lit - ce qui rend la propriété du symbole conjugal encore plus éloquente. 
figuré au récit du poète ${ }^{62}$, dans des conditions qui en éclairent le sens. C'est ici d'autant plus indubitable que le symbolisme du chien pour désigner Ulysse s'inscrit dans une mise en œuvre plus large, et que la sorte de "programme " illustré posé par Pénélope sur les vêtements dont elle habilla son mari prêt à partir, se trouve en rapport intime avec le choix décisif d'Ulysse de rentrer auprès d'elle.

\section{L'image du chien dans le sjstème poétique}

Il faut en effet approfondir l'affinité qui existe entre le maître d'Ithaque et l'animal proprement « domestique » qu'est le chien. L'image d'une adéquation entre l'un et l'autre est poussée si loin qu'elle est utilisée comme procédé poétique pout souligner l'enjeu essentiel du retour d'Ulysse, à savoir l'acceptation de sa mort. Qu'on songe d'abord au "vrai » chien Argos, soumis au vieillissement et à la mort - à telle enseigne qu'il meurt pour ainsi dire sous nos yeux (XVII, v. 290 sq.) : tout se passe comme si cette bête, dont le texte ne souffle mot par ailleurs, n'existait dans le poème que comme "double » de son maître, dont il partage la déchéance physique ${ }^{63}$; il semble être à tel point son représentant que (dirait-on) il lui cède la place pour garder la maison en s'effaçant dès son retour. À l'opposé de ce chien en chair et en os - bien individualisé (aussi a-t-il un nom), qui vieillit et qui meurt -, on rencontre les chiens anonymes, en or et en argent, qu'Héphaïstos précisément (et il n'est pas indifférent qu'on le rencontre ici) offrit au roi Alkinoos : ces créatures divines, faites d'une matière incorruptible, sont quant à elles « immortelles et éternellement jeunes » (VII, 94). Or ce couple d'adjec-

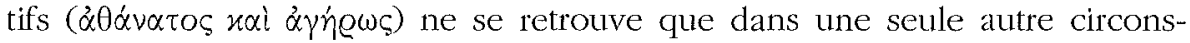
tance (mais alors, avec quelle insistance !) : quand il s'agit de l'immortalité et de l'éternelle jeunesse qui séparent la condition de Calypso de celle d'Ulysse.

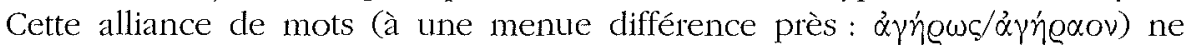
revient pas dans ce contexte à moins de quatre reprises (V, 136; 218; VII, 257; XXIII, 336). Toutefois comme on sait, Ulysse refuse ${ }^{64}$ la proposition que lui fait la déesse de partager ces privilèges, en lui disant avec toute la délicatesse possible que, certes, Pénélope n'est pas «immortelle et éternellement jeune » comme elle $(V, 218)$, mais qu'il préfère néanmoins rentrer chez lui. Il semble donc indiqué de comprendre que les chiens immortels de la «contre-

${ }^{62}$ Comme on le voit, je fais comme si le texte homérique était un texte comme un autre. Ce n'est pas que j'ignore les innombrables problèmes spécifiques qu'il pose. C'est parce que j'estime qu'une approche littéraire candide possède aussi sa légitimité - d'autant plus qu'elle peut faire rebondir le débat homérique sur de nouveaux frais.

${ }^{63}$ Il demeure entendu que le "corps d'Ulysse " revêt plusieurs apparences : cf: J. PEIGNEY, «Les deux corps d'Ulysse. Une construction possible pour l'Odyssée, chants XIII à XXIII ", LEC 53 (1985), p. 341-54.

G4 Cf. J.-P. VERNANT, «Le refus d'Ulysse », in TR 3 (1982), p. 13-18. Il faut encore souligner que l'ouverture du chant $\mathrm{V}$ se fait sur Eos et Tithon, autre couple disparate. 
Ithaque » qu'est la Phéacie ${ }^{\text {is }}$, se réfèrent aussi à Ulysse - en son choix le plus déterminant: celui de l'humanité qui implique la mortalité. En ce que ces animaux merveilleux ne sont pas autrement "décrits», en ce qu'il n'est plus ailleurs question d'eux dans le poème, quelle utilité pourraient-ils bien avoir, que de servir à répercuter dans l'imagerie canine le contraste entre d'une part le personnage d'exception qui fraie avec les dieux et se voit offrir immortalité et jeunesse, et de l'autre "l'homme » (áv $\delta \varrho \alpha$ n'est-il pas le tout premier mot du poème : $O d$. I, 1 ?) qui choisit de rester assujetti au vieillissement et à la mort, dont on constate les ravages sur son chien Argos ? Ainsi la valeur symbolique du chien dans l'odyssée se trouve-t-elle élargie à la dimension de procédé poétique destiné à souligner la signification, cruciale pour la substitution des hommes aux héros, de la décision d'Ulysse ${ }^{66}$.

On voit donc que le chien de la broche vient s'intégrer à un processus narratif qui sous-tend le poème tout entier. Mais lui n'est pas concerné par l'opposition entre la vie périssable et l'éternelle jeunesse. Ce chien placé par Pénélope en évidence sur le manteau d'Ulysse dès avant son départ figure un troisième terme: il fixe par avance (sous une forme en quelque manière désincarnée et soustraite à tout contexte spatial et temporel ${ }^{67}$ par le symbolisme animal) la juste vengeance dont cette miniature ciselée précède et transcende l'accomplissement effectif. Cette représentation artistique est donc en rapport avec une scène essentielle du poème. Cependant au lieu d'offrir un correspondant global de l'odyssée entière, la broche se contente de proposer un équivalent transposé d'un épisode du poème, et donc de s'en tenir à une partie de la diégèse qui est représentable, et qui ne remet pas en cause les relations des hommes et des dieux. C'est pourquoi cette œuvre, qui certes suscite l'admiration (v. 229) n'a pourtant pas la perfection abstraite d'être simplement $\theta \alpha \tilde{\mu} \mu \alpha$, comme la pure merveille qu'est le bouclier divin d'Achille (Il. XVIII, 549) : aussi bien sa fabrication n'est-elle pas attribuée à Héphaïstos.

Jusqu'à présent tout se passe donc comme si les œuvres humaines (en particulier les ouvrages ou présents féminins que nous venons de considérer : étoffes, manteau ou broche) remplissaient dans les deux épopées une fonction symbolique, mais qu'il appartenait à l'œuvre divine seule de "réfléchir » celle du poète, en renvoyant sur le bouclier une image de l'xliade où

${ }^{65}$ Sur l'épisode à Schérie, vu comme un premier « retour d’Ulysse " à soi, $c f$. J. PEIGNEY, «La Phéacie, terre poétique », in F. JouAN, B. DeforGe, Peuples el pays mytbiques, Paris, 1987, p. 4755.

6r Sans doute est-il significatif qu'Achille ait été placé devant un choix analogue : une vie brève et glorieuse, ou longue mais obscure.

${ }^{67}$ On retrouve la même préoccupation de situer l'action du héros sur un fond qui échappe à la succession chronologique quand on considère la fête d'Apollon dont il est question en $\mathrm{Od}$. XX, 156 et XXI, 258. Si la broche fixe dans l'éternité de l'or le symbolisme animal pour représenter le monde lumain, la fête d'Apollon quant à elle intègre positivement la vengeance humaine dans la périodicité du rituel qui renvoie aux temps mythiques : c/. AUBriot, "Autour de quelques fêtes " (cf. supra, n. 48), p. 70-73. 
ombres et lumières ont échangé leurs rôles, comme sur un négatif photographique. Ainsi, personne d'autre que le dieu n'est imaginé posséder la distance suffisante pour prendre à son compte un face à face plastique de la vie et de la mort, parallèle au face à face poétique qu'en présente l'Iliade, et pouvoirles contempler d'assez haut pour en reconsidérer les rapports.

Que cette interprétation soit réservée aux réalisations d'Héphaïstos se voit confirmé par quelques brèves considérations complémentaires, où il apparaît que l'Odyssée vient décidément corroborer ce que le bouclier d'Achille dans l'lliade induisait à conclure.

\subsection{Les oeuvres du dieu et la signification générale des poèmes : l'ui'ne}

\section{Le bouclier et la mont des béros}

Déjà la pseudo-description du bouclier d'Achille apparaissait (comme nous l'avons dit plus haut) non seulement comme une mise en abyme du monde, mais comme une réverbération symétrique du poème lui-même ${ }^{68}$. Cependant il n'a pas encore été rappelé que sur ce bouclier figurent deux images ou reflets de la mort: le Trépas sanglant qui précipite chez Hadès, et de possibles onxoús (enclos funéraires hérö̈ques) ${ }^{69}$ laissant entrevoir d'autres perspectives. Ces deux vignettes (énigmatiques en leur brièveté) semblent évoquer le texte d'Hésiode ${ }^{70}$ selon qui, des héros qui périrent sous les murs de Thèbes ou de Troie, les uns disparurent chez Hadès, enveloppés par « la mort qui tout achève», tandis que d'autres s'en furent «aux confins de la terre $»^{71}$. Toutefois le texte homérique est plus qu'allusif concernant 1'hypothèse d'une destination fortunée pour quelques-uns des héros, et laisse cette espérance dans un flou qui pour ainsi dire l'étouffe. Cela pourrait suggérer

68 L'lliade présente encore quelques ouvres d'Héphaïstos : cf. M. DFLCOURT, Héphaïstos ou la légencle du magicien, paris, 1957, p. 48 sq.

69) Il. XVIII, 535-41; 587-9. Cf. AUBRIOT, «Imago Iliadis» (supra, n. 3), p. 19-21 et 33-39.

${ }^{70}$ Hés., T.J, 156-73: «Zeus, fils de Cronos, en créa encore une quatrième (race) sur la glèbe

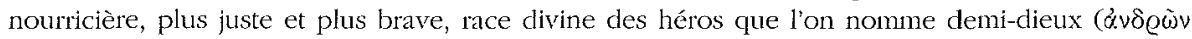

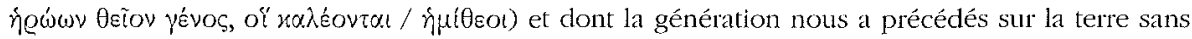
limites. Ceux-là périrent dans la dure guerre et dans la mêlée douloureuse, les uns devant les

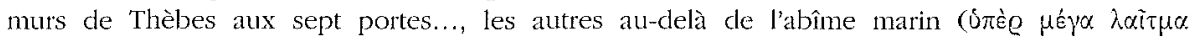
$\theta \alpha \lambda \dot{\alpha} \sigma \sigma \eta \varsigma)$ à Troie, où la guerre les avait conduits sur des vaisseaux, pour Hélène aux beaux cheveux, et où la mort, qui tout achève, les enveloppa. À d'autres enfin Zeus, fils de Cronos et père des dieux, a donné une existence et une demeure éloignées des hommes, en les établissant aux confins de la terre. C'est là qu'ils habitent, le coeur libre de soucis, dans les Îles des Bienheureux, aux bords des tourbillons profonds de l'Océan, héros fortunés, pour qui le sol fécond porte trois fois l'an une florissante et douce récolte» (trad. Mazon). Faire ce rapprochement n'implique pas la suggestion qu'il y aurait un écho délibéré d'Hésiode dans Homère. Sans vouloir prendre parti pour le moment sur la délicate question de l'antériorité de l'un par rapport à l'autre (cf. A. BALlabRigA, «La Question Homérique : pour une réouverture du débat », REG 103 [1990], p. 16-29), je constate simplement une ressemblance.

${ }^{71}$ HÉs., $T / .$, 168. Le texte d'Hésiode non plus, à vrai dire, n'est pas limpide, et n'explique pas au juste lesquels de ces héros sont admis aux îles des Bienheureux. 
une certaine réserve devant la possibilité d'accéder à un au-delà moins désespérant que les demeures d'Hadès. De plus le bouclier fabriqué par Héphaïstos, en tant qu'il est ouvré sur la demande de Thétis, est certes un gage de la semi-divinité d'Achille; mais dans le même temps, désigné comme ne pouvant éviter la mort au héros (II. XVIII, 464-466), il est une preuve qu'il n'existe plus de demi-dieux immortels. En somme les représentations qu'il porte, tout autant que les circonstances accompagnant sa fabrication, suggèrent d'en faire un témoignage du gouffre désormais ouvert entre les hommes et les dieux ${ }^{72}$, après la disparition de la race intermédiaire qu'était celle des héros. Si cette manière d'appréhender le texte a quelque fondement, le Boiteux viendrait apporter sa caution au "plan de Zeus » (qui se réalise dans l'Tliade) de mettre fin à l'existence de cette race héroïque ${ }^{73}$, et les ouvrages qu'il fabrique seraient ainsi en relation étroite avec le sens profond du poème - qui concerne le rapport (en cours d'établissement définitif, selon les desseins de Zeus) entre la vie et la mort des hommes. On comprendrait donc que le texte esquisse une sorte de parallèle entre le dieu et le poète, entre l'œuvre quasi démiurgique du premier, et la création poétique du second.

\section{"L'urne - cercueil » pour les «os blancs» d'Achille et de Patrocle}

Mais quelle serait alors la place d'Héphaïstos dans l'Odyssée où il est plutôt question des tribulations de "l'homme " (en la personne d'Ulysse), une fois presque achevée l'extinction des héros ? On pourrait s'attendre à y trouver ce dieu en retrait. Or il est remarquable que la seconde des épopées homériques permette d'approfondir la réflexion dans le sens même des investigations entamées. En effet, entre les quelques ouvres d'art réalisées par ce dieu qui sont signalées dans l'Odyssée, la plupart semblent étrangères au monde de la mortalité : deux sont des présents offerts à des personnages situés hors de l'humanité (ce sont les chiens de ce monde intermédiaire qu'est la Phéacie, et le cratère donné au héros Ménélas, appelé pour sa part à se rendre dans les îles des Bienheureux parce qu'il est le gendre de Zeus ${ }^{75}$ ); une autre, admirée par les Immortels dans le chant médian de Démodocos, est le filet merveil-

\footnotetext{
72 Gouffre dont le personnage d'Achille est un symbole, comme j'ai tenté de l'illustrer dans «Humanité et divinité » (cf. supra, n. 23).

${ }^{73}$ Gf. supra, p. 51 et n. 25.

74 Il. XXIII, 252; Od. XXIV, 76.

${ }^{75} \mathrm{Od}$. IV, 569 . Ce cratère remis par le dieu au héros est ensuite par lui offert à Télémaque transfert qui, par l'intermédiaire de l'époux d'Hélène, le fait passer du dieu à l'homme ( $O d$. IV, 614-9, et XV, 114-9). Que les cuvres d'Héphaïstos possèdent ainsi un caractère de charnière entre les deux mondes se laisse apercevoir à travers d'autres indices. En particulier, très significative apparaît la métamorphose d'Ulysse ressortant du bain qu'il s'est donné lui-même " dans les courants du fleuve (Od. VI, 216) de Phéacie : "Voici qu'Athéna... le faisant apparaitre et plus grand et plus fort, déroulait de son front des boucles de cheveux aux reflets d'Hyacinthe; tel un artiste habile, instruil par Héphaïstos et Pallas Athéna de toutes leurs recettes, coule en or sur argent un chef-d'ouvre de grâce : telle Athéna versait la grâce sur la tête et le buste d'Ulysse » ( $\mathrm{Od}$. VI, 228-35). Comme ne manque pas de le remarquer Nausicaa, cet "homme » (ávńe, v. 241) ressemble désomais aux dieux (0єoioı ěouxe, v. 243). Il s'impose de relever la corrélation entre cette apparence divine et le travail d'Héphaïstos.
} 
leux forgé pour enserrer Arès et Aphrodite (qui donc concerne une affaire exclusivement divine ${ }^{76}$ ). Par conséquent on pourrait avoir l'impression que les réalisations du dieu mentionnées dans l'Odyssée confinent tacitement les mortels dans un monde subalterne (en ce qu'elles sont fabriquées pour des destinataires de nature supérieure), mais qu'elles n'ont rien à voir avec l'articulation entre hommes et dieux, ni avec le propos du poète.

Pourtant il en est une qui, malgré la discrétion avec laquelle elle est citée (sans l'amorce de la moindre description et pour ainsi dire en litote), ramène à cette question que je crois essentielle à la poésie homérique : celle du terme définitif représenté par la mort. C'est "l'urne » d'or qui doit contenir les ossements mêlés de l'homme et du demi-dieu : Patrocle rejoint par Achille. À elle seule, cette destination suffirait à alerter sur la charnière entre héros et homme. Mais il se trouve de surcroît que cette « urne», comme disent nos traductions, n'en est pas une. Exempte de toute décoration, donc apparemment lisse (ce qui constitue une forme d'abstraction), elle revêt de plus une forme incertaine : nommée tour à tour cercueil, amphore ou phiale, elle se dérobe à toute représentation réaliste, ce qui fait d'elle une «idée » de récipient plutôt qu'un vase véritable. En somme, elle aussi (comme le bouclier mais d'une autre manière) n'est rien d'autre que «cosa mentale». Il est d'autant plus important de le concevoir que c'est la seule œuvre d'Héphaïstos qu'on retrouve d'un poème à l'autre. Elle apparaît (comme on peut s'y attendre) dans deux passages où il est question des morts et de l'au-delà : les funérailles de Patrocle et la seconde Nekyia de l'Odyssée. Ce qui était moins prévisible, c'est que sa mention, les deux fois, soit mise dans la bouche d'un mort (Patrocle dans le songe d'Achille, Agamemnon aux Enfers); en sorte que cette «urne » a toutes les apparences d'un Graal surnaturel. L'Iliade en parlait sans indications sur son origine : "L'amphore d'or que t'a procurée ta véné-

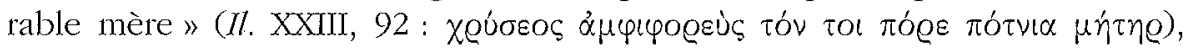
disait Patrocle à son compagnon en lui demandant d'y déposer ses cendres dans l'attente de sa propre mort ${ }^{77}$. Il faut attendre le dernier chant de l'Odyssée pour découvrir simultanément (et $i n$ extremis, au dénouement ultime des

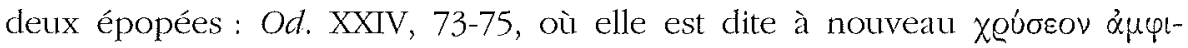
$\varphi \circ \varrho \tilde{\eta} \alpha$ ), à la fois que Thétis la donnait pour un présent de Dionysos, et qu'elle était un ouvrage "de l'illustre Héphaïstos ». Or ces deux dieux, avant d'être réconfortés par la mère d'Achille, avaient connu une sorte de persécution (qui

7 Od. VIII, 266 sq. Affaire divine, certes, mais en rapport avec mariage et adultère, questions centrales pour l'Odyssée. Sur les ouvrages d'Héphaïstos mentionnés dans l'Odyssée, cf. Delcouit' supra, n. 68.

77 Et un peu plus loin (Il. XXIII, 243), Achille donnait l'ordre de placer les restes de son

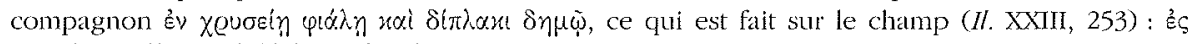

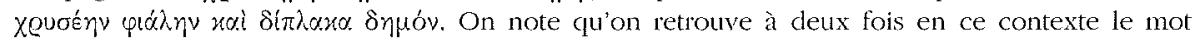
$\delta i ́ \pi \lambda \alpha \alpha$, qui avait servi pour les deux étoffes tissées par Hélène et Andromaque - et que son emploi (si l'on s'en tenait à une interprétation réaliste) serait tout aussi obscur ici que là. Cette récurrence semble bien confirmer que cette «double " appartenance, de la pièce d'étoffe comme du dernier séjour, vise la double nature, humaine et héroïque. 
les avait fait choir dans la mer où la déesse les avait " accueillis " ${ }^{78}$ ), et avaient tâté de la souffrance, ce qui d'ordinaire est le lot des hommes. Ce don acquiert donc une signification particulière et les précisions tardives sur sa provenance et sa transmission divines, qui le font circuler entre des mains de dieux ayant pour ainsi dire côtoyé la mort, le montrent bien fait pour renfermer mêlés - préservés de toute altération, mais réduits en poussière les ossements du demi-dieu violent ( $(\alpha \alpha \tau \varepsilon \varrho o \varsigma)$ qu'était Achille aussi bien que

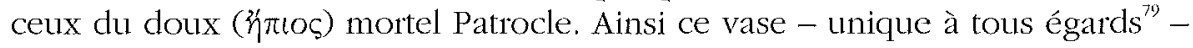
voit-il son usage parfaitement adapté au nom dont le désignent les morts qui en parlent pour évoquer le mélange des ossements, nom ( $\alpha \mu \varphi \varphi$ pogúc) qui peut suggérer non seulement une double préhension, mais ici, en plus, un double contenu ${ }^{80}$ : les restes, confondus dans la mort, des deux natures, héroïque et humaine.

On ne peut donc en rester à l'opinion selon laquelle le rôle d'Héphaïstos et de ses créations dans l'Odyssée serait purement pittoresque, et relèverait de ce qu'on appelle le «merveilleux épique». Bien au contraire, cette ouvre sans pareille qu'est « l'urne » d'or nous replonge au cour même et du sens, et de la composition des poèmes. Traitée dans l'lliade de "cercueil » (oogós Il. XXIII, 91), elle ne peut que ramener sans ambiguïté au thème de la mort. Mais désignée dans les deux épopées comme une "amphore d'or » (Il. XXIII, 92 et $O d$. XXIV, 74), elle laisse présumer que l'art, ou en tout cas le métal précieux et incorruptible, peut offrir un réceptacle brillant et inaltérable même à des restes détruits par la mort et blanchis par le feu. En sorte que cette œuvre d'Héphaïstos semble bien constituer un lien délibérément conçu dans "les savants pensers" du poète, cette fois - pour rappeler qu'aboutissent finalement au même résultat la semi-divinité d'Achille (dont l'Tliade fait

${ }^{78}$ Chacun des deux dieux (Héphaïstos et Dionysos) rappelle que «Thétis (l')accueillit en son

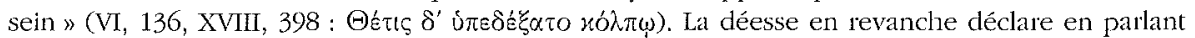

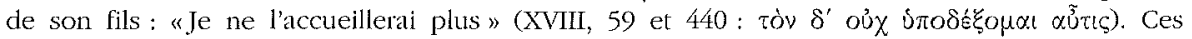
hénistiches qui se répondent suggèrent la possibilité de considérer les deux dieux persécutés et le héros en quelque sorte comme des demi-frères. Mais alors que l'accueil de Thétis détermina pour les deux premiers une impulsion donnée à leur réalisation comme dieux, le cas d'Achille se présente à l'inverse : c'est bien parce qu'il est mortel, et qu'il va mourir, qu'elle ne pourra plus l'«accueillir»».

79) On n'en connaît pas d'autre dans les deux épopées, encore moins pour mélanger les restes différents de guerriers qui ne meurent pas en même temps. À cela s'ajoute que cette ó $\mu_{\grave{n}}$

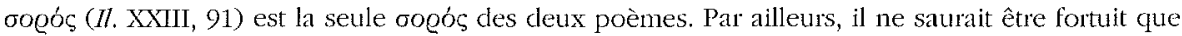
l'Odjssée comporte, en sa fin, une relation circonstanciéc des funérailles d'Achille accompagnée de rappels formels ( $O d$. XXIV, $40=I l$. XVI, 776) ou plus lâches, mais nets (les vers $38-94$ en regorgent), qui relient explicitement cette cérémonie à celle des funérailles de Patrocle dans l'lliade. Il ne saurait être indifférent non plus que cette relation soit faite avec recueillement par l'ombre d'Agamemnon censée parler à l'ombre d'Achille, en un dialogue bien éloigné de la querelle qui ouvre l'lliade - ce qui indique un autre lien, cette fois entre la fin du second poème et le début du premier.

${ }^{80}$ Cette «urne " aussi se trouve donc être un vase du mélange (comme le «cratère » à deux métaux donné par Ménélas à Télémaque : cf. sutpra, n. 75), mais dans un contexte de réunion dans la mort, et non pas de banquet joyeux et partagé. 
sentir le caractère fallacieux), et la mortalité partagée par tous les hommes: par Patrocle, sans doute, mais aussi particulièrement en cette fin de l'Odyssée, par «l'homme» par excellence qu'est Ulysse, qui avait sciemment refusé d'être "immortel et jeune à tout jamais »" et dont la mort en sa vieillesse est annoncée par Tirésias au milieu du poème (Od. XI, 134-137). Ainsi ce vase à la fois divin et funéraire pourrait-il, en même temps qu'il réunit les cendres des deux compagnons, mêler le sens de l'thiade (la fin des héros) à celui de l'Odyssée (l'avènement de l'homme), tout comme il assure un lien textuel entre les deux poèmes ${ }^{82}$.

Récapitulons brièvement. Si, pour s'interroger sur les représentations artistiques chez Homère, on en revient au chassé-croisé qui met en relation les ouvres métalliques d'Héphaïstos et les figurations dessinées par les femmes sur les vêtements, on est amené à des constatations dont les conséquences ne sont rien moins que superficielles.

Sans doute les réalisations des femmes sont-elles, comme celles du dieu, en correspondance avec la narration du poète. Toutefois les conditions sont différentes. Les travaux d'Hélène et d'Andromaque se contentent de dégager les composantes de l'expression poétique de l'Iliade en distribuant séparément ses deux registres sur chacune des deux pièces d'étoffe. Pour l'Odyssée, la broche donnée par Pénélope synthétise dans une allégorie concrète, imagée et symbolique ce qui fait l'essence même du retour de l'époux-roi : la vengeance et la réintégration dans les prérogatives qui lui appartiennent. Ces ouvrages féminins supposent déjà entre poésie et représentations plastiques des combinaisons dont l'intérêt n'est pas négligeable. Mais les œuvres du dieu quant à elles touchent d'une manière plus profonde à la substance même de la poésie homérique. En suggérant la béance ouverte entre héros et hommes, (et en même temps leur destination identique vers le trépas), elles figurent (le bouclier) ou abritent (l'urne) parallèlement le crépuscule des héros entamé dans l'Iliade et l'aube du règne de l'homme inauguré dans l'Odyssée : par là, non seulement elles travaillent la même matière que le poète, mais encore elles réunissent les thèmes des deux épopées. Cette réunion ne pouvait s'opérer qu'à la hauteur d'un dieu qui, capable de considérer les vicissitudes humaines d'une vue cavalière, hors du temps, et d'embrasser à la même distance les vanités de la grandeur hérö̈que et les efforts de l'endurance humaine, soit en mesure de montrer avec autorité que la seule vraie supério-

81 Il est symptomatique que, dans la demière réplique de la seconde Nekyia qui ouvre le chant XXTV de l'Odyssée, l'ombre d'Agamemnon s'adresse à Ulysse au vocatif, comme s'il était présent parmi les morts (v. 492-202).

${ }^{82}$ Sans doute est-il significatif que la "remontée » jusqu'à l'origine de ce vase survienne à l'extrêne fin de l'Odyssée, au moment où Ulysse va retrouver, avec son père, la dynastie familiale : preuve qu'il est définitivement étranger à la destinée héroïque, puisqu'il s'intègre à la succession des générations et de leurs âges successifs : cf. R. SCODEL, "The Achaean Wall and the Myth of Destruction (II. VII \& XII) », HSPb 86 (1982), p. 33-50. 
rité appartient à l'art, reconnu désormais comme le lien privilégié entre mortels et dieux, l'unique participation ${ }^{83}$ possible de l'homme à la divinité.

Danièle AuBrioT

26, place de la République

F - 80290 POIX-DE-PICARDIE

83 Participation à double sens, car la musique et le chant passent des divinités aux hommes par l'inspiration reçue du poète; mais aussi réciproquement, ils retournent des hommes aux divinités lors des célébrations qui sont organisées en leur honneur : dans ces fêtes, les fidèles (exécutants du rite) et les dieux (récipiendaires du culte) communient au plaisir partagé qu'ils goûtent : cf. Aubkio'T, "Autour de quelques fêtes » (supra, n. 48), p. 78-80. 